\title{
Strain Induced Vertical and Lateral Correlations in Quantum Dot Superlattices
}

\author{
V. Holý, ${ }^{1,2}$ G. Springholz, ${ }^{1, *}$ M. Pinczolits, ${ }^{1}$ and G. Bauer ${ }^{1}$ \\ ${ }^{1}$ Institut fuer Halbleiterphysik, Johannes Kepler Universität, A-4040 Linz, Austria \\ ${ }^{2}$ Laboratory for Thin Films and Nanostructures, Masaryk University, 61137 Brno, Czech Republic
}

(Received 17 February 1999)

\begin{abstract}
Vertical and lateral ordering of quantum dots in superlattices is shown to be determined by the elastic anisotropy of the matrix material and by the growth orientation. For large anisotropies, complicated dot stacking sequences with correlations inclined to the growth axis may be formed, and the lateral ordering tendency is much stronger than for isotropic materials.
\end{abstract}

PACS numbers: 68.55.Jk, 68.65.+g

Much effort has been devoted to the fabrication of semiconductor nanostructures because of their great potential for semiconductor devices. Apart from processing techniques involving lithography and etching, the direct growth of nanostructures has evolved as a promising new approach. It is based on the tendency of strained epitaxial layers to spontaneously form coherent three dimensional (3D) islands after completion of the two dimensional (2D) wetting layer. In multilayer structures, the buried dots tend to influence the nucleation in subsequent layers [1-4]. Based on a one dimensional isotropic model, Tersoff et al. [4] have proposed that the resulting vertical alignment of the dots in subsequent layers can lead to a lateral ordering as well. Although the vertical correlation can be nearly perfect [1-3], experimentally, the lateral ordering was found to be much less pronounced [46]. In addition, apart from the vertical dot alignment in $\mathrm{Ge} / \mathrm{Si}$ and InAs/GaAs superlattices, an anticorrelation of the dots on subsequent interfaces was observed for submonolayer $\mathrm{CdZnSe}$ islands embedded in $\mathrm{ZnSe}$ [7], which was explained by considering the elastic interactions as a function of spacer thickness [8]. Even more, for $\mathrm{PbSe}$ dot superlattices we have recently reported a fcc-like $A B C A B C$ vertical dot stacking sequence [9].

It is the purpose of this Letter to show that all forms of vertical and lateral correlations in self-assembled quantum dot superlattices can be explained by taking into account the elastic anisotropy of the materials. In these superlattices, dot correlations are induced by the interaction of the dots via their elastic strain fields. Above the buried islands, the elastic energy distribution on the surface exhibits pronounced minima and maxima in the lateral directions. This leads to a diffusional bias of the deposited adatoms and to a preferential nucleation of the dots at the local strain minima on the surface, which are linked to the dot positions in the previous layer. Here it is shown that depending on the elastic properties and the growth orientation, a variety of dot stacking types may be formed, and that a most effective lateral ordering occurs for materials with high elastic anisotropy.

For a given nonuniform strain distribution $\epsilon_{i j}(\mathbf{r})(i, j=$ $x, y, z)$ within a strained dot superlattice, the elastic en- ergy density on the surface at $z=0$ is given by $E(x, y)=$ $1 /\left.2 c_{i j k l} \boldsymbol{\epsilon}_{k l}(\mathbf{r}) \epsilon_{i j}(\mathbf{r})\right|_{z=0}$, where $c_{i j k l}$ are the elastic constants in the coordinate system defined by the sample surface. Because of the lattice distortions around the buried dots, the strain distribution on the wetting layer is given by the superposition of the homogeneous lattice-mismatch strain $\epsilon_{i j}^{(0)}$ and the contribution $\epsilon_{i j}^{(1)}(\mathbf{r})$ of the buried islands. For an infinite elastically anisotropic medium, the strain distribution $\Delta \epsilon_{i j}(\mathbf{r})$ induced by a buried point stress source has been solved analytically [10].

To obtain the solution for a semi-infinite medium, we have solved the equilibrium equations

$$
\frac{\partial \Delta \sigma_{j k}}{\partial x_{k}}=\Delta G \frac{\partial \delta(\mathbf{r}-\mathbf{R})}{\partial x_{j}}, \quad j=x, y, z,
$$

where $\Delta \sigma_{i j}$ is the stress tensor due to an elementary stress source $\Delta G=\epsilon_{0} \Delta V\left(c_{11}^{c}+2 c_{12}^{c}\right)$ [11] in a point $\mathbf{R} . \Delta V$ is the infinitesimal volume of the stress source, $\epsilon_{0}$ the lattice mismatch between source and surrounding crystal, and $c_{\alpha \beta}^{c}$ the elastic constants in the crystallographic coordinate system. The equilibrium equations were solved by the Fourier method [12] with the boundary condition of an unconstrained free surface $\Delta \sigma_{j z}=\left.0\right|_{z=0}$. The strain $\epsilon_{i j}^{(1)}(\mathbf{r})$ caused by a buried island can then be calculated by a summation of the $\Delta \epsilon_{i j}(\mathbf{r})$ over the whole island volume $V_{\text {island }}$. Outside of the highly strained quantum dot regions, the continuum elasticity calculations are usually in good agreement with atomistic calculations [13].

Since we are interested mainly in generic effects, we restrict our analysis to the case where the lateral and vertical extent of the islands can be neglected, and the whole stress source is thought to be concentrated in a single point $\mathbf{R}$. This describes the actual situation rather well as long as the thickness of the spacer layer is about 3 times larger than the island size. Then, the strain distribution on the surface of the wetting layer is

$$
\epsilon_{i j}(\mathbf{r})=\epsilon_{i j}^{(0)}+\Delta \epsilon_{i j}(\mathbf{r}) V_{\text {island }} / \Delta V .
$$

Using Eq. (1), the relative change of the strain energy on the surface above a buried island is given by

$$
\rho(x, y)=\left[E_{1}(\mathbf{r})-E_{0}\right] /\left.E_{0}\right|_{z=0},
$$


where $E_{0}$ is the constant energy density in the wetting layer due to the misfit strain, and $E_{1}$ is the energy density with a buried island underneath. Because of this renormalization, $\rho$ does not depend on the lattice mismatch between island and matrix material. However, $\rho$ still scales linearly with the island volume, which varies for different material systems and growth conditions. To allow a comparison, we have fixed the island volume to $7200 \mathrm{~nm}^{3}$ (pyramid of $20 \mathrm{~nm}$ height and $50 \mathrm{~nm}$ base length). In addition, the magnitude of the $\rho$ variations decreases inversely with the cube of the spacer thickness $h$. In the following, $h$ was fixed to $50 \mathrm{~nm}$, but it is emphasized that the shapes of the energy density contours are invariant when plotted over the dimensionless coordinates $x / h$ and $y / h$.

From our calculations it turns out that the elastic energy distributions are determined by two key parameters, namely, (1) the elastic anisotropy of the matrix material, and (2) the surface orientation. For cubic materials, the elastic anisotropy can be characterized by the anisotropy ratio $A=2 c_{44}^{c} /\left(c_{11}^{c}-c_{12}^{c}\right)$. It is roughly equal to the ratio between the Young's modulus $E$ along the $\langle 111\rangle$ and the $\langle 001\rangle$ directions. These are the directions where $E$ has its extremal values. For isotropic materials $A=1$, i.e., $E$ does not depend on the direction of the applied extensional forces. For the group IV, the III-V and II-VI semiconductors with diamond or zinc-blende structure and with the chemical bonds along $\langle 111\rangle$, the $\langle 111\rangle$ directions are the elastically hard directions, and the $\langle 001\rangle$ directions the soft directions $(A>1)$. The anisotropy is largest for the II-VI compounds, with $A=2.04$ for $\mathrm{ZnTe}$ and 2.53 for $\mathrm{ZnS}$. For $\mathrm{C}, \mathrm{Si}$, and $\mathrm{Ge}, A$ increases from 1.21, 1.56, to 1.64 , respectively; and for the III-V compounds, $A$ ranges from 1.83 for GaAs to 2.08 for InAs. For rock salt materials, however, the next nearest neighbors are along $\langle 001\rangle$.
Thus, $A<1$ and $\langle 001\rangle$ are the hard directions and $\langle 111\rangle$ the soft directions. For the IV-VI semiconductors, the elastic anisotropy is particularly large, with $A=0.18,0.27$, and 0.51 for $\mathrm{SnTe}, \mathrm{PbTe}$, and $\mathrm{PbS}$, respectively.

To characterize the elastic interactions between the dots on the surface and the buried dots, we have calculated the elastic energy distributions for different material systems and growth directions. Representative $\rho(x, y)$ contour plots for the (001), (111), and (113) surfaces are shown in Fig. 1, together with cross sectional profiles $\rho(r)$ for $\mathrm{PbTe}, \mathrm{PbS}, \mathrm{Si}, \mathrm{GaAs}$, and $\mathrm{ZnSe}$ as matrix materials. For elastically isotropic spacer layers (dashed lines), the energy minimum is always in the origin of $\rho(x, y)$, independent of the surface orientation. However, the elastic anisotropy changes both the depth and the position of the energy minima; in particular, the $\rho(x, y)$ distributions are qualitatively different for the growth along the elastically hard or soft directions.

For the growth along the hard direction ([111] for diamond and zinc blende and [001] for rock salt materials), the depth of the energy minimum strongly increases with increasing anisotropy, but its position above the buried dot remains unchanged. The opposite behavior is observed for growth along the soft direction ([001] for diamond and zinc blende and [111] for the IV-VI materials); i.e., the depth of the minimum decreases as the anisotropy increases. Even more, when the anisotropy ratio exceeds a critical value, the central minimum is replaced by several side minima in the $\rho(x, y)$ distributions. For the (001) surface and $A>1.5, \rho(x, y)$ exhibits four minima along the $\langle 110\rangle$ directions. For $\mathrm{Si}$ and GaAs these minima are quite shallow, but become pronounced for higher anisotropies (see ZnSe, Fig. 1). For the (111) surface and $A<0.6$ $(\mathrm{PbS}, \mathrm{PbTe}$, etc.), three deep side minima occur along

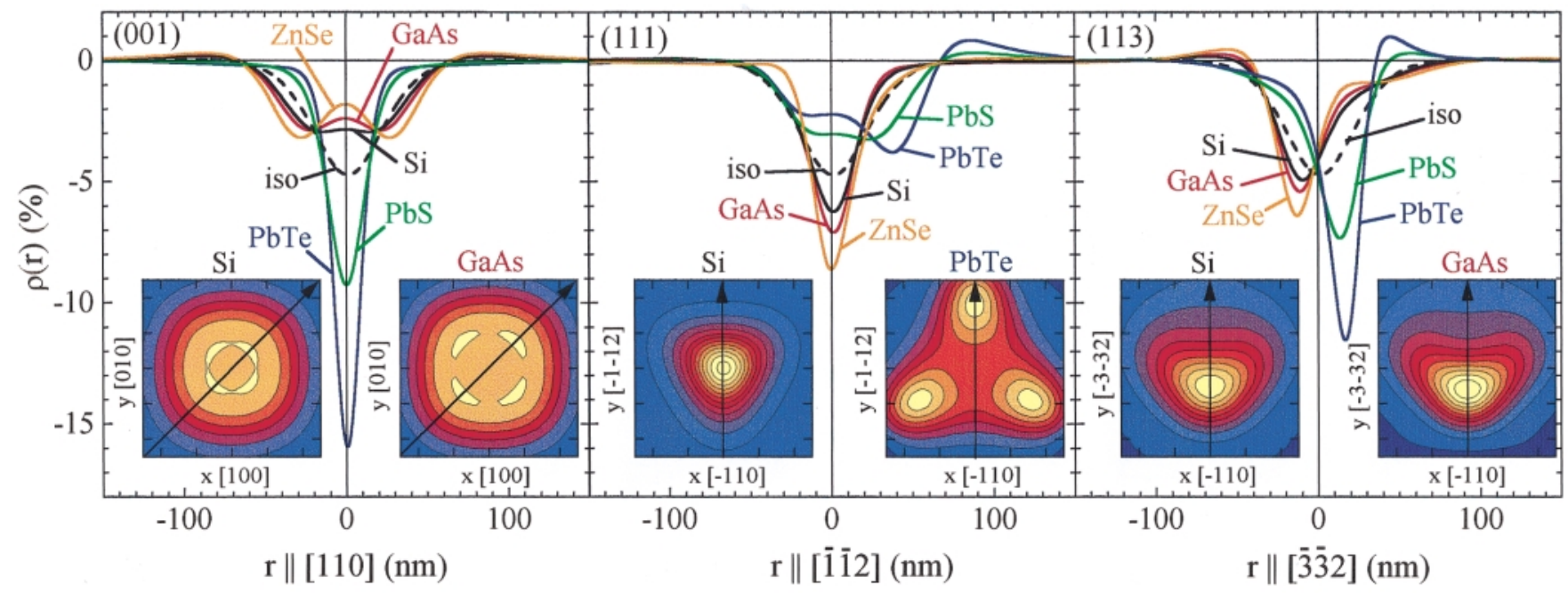

FIG. 1 (color). Normalized elastic energy density $\rho(x, y)$ on the surface above a strained self-assembled quantum dot at $50 \mathrm{~nm}$ below the surface for various matrix materials and for the (001), (111), and (113) surface orientations (from left to right). The curves represent cross sections of $\rho$ in the directions indicated by the arrows in the insets. Insets: 2D contour plots with $-50 \leq(x, y) \leq+50 \mathrm{~nm}$ and with a $0.5 \%$ step width between contours. The yellow areas correspond to the minima of $\rho$. 
the $\langle\overline{11} 2\rangle$ directions. Such side minima are a general feature for all surface orientations close to a soft direction, provided that the elastic anisotropy is sufficiently large. Although otherwise only one $\rho$ minimum appears, it is not necessarily located above the buried dot but can be displaced in a lateral direction. As shown in Fig. 1, for (113) $\mathrm{Si}$ and $\mathrm{GaAs}(A>1)$ the minimum is shifted in the

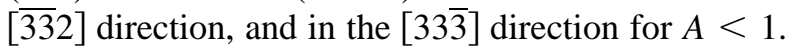

The chemical trends are summarized in Fig. 2 for the (001) and (111) surface orientations. The top panel shows the energy density $\rho(0)$ at $x, y=0$ (full dots) and $\rho_{\text {min }}$ at the side minima (open circles) versus anisotropy ratio. Clearly, the more $A$ deviates from unity, the more pronounced is the difference between the center and the subsidiary minima. To characterize the minima positions, we define $\alpha$ as the angle between surface normal and the vector from the buried island to the energy minimum on the surface. As shown in the lower panel of Fig. 2, this angle changes continuously with varying anisotropy. For the (001) surface, $\alpha$ increases linearly with increasing elastic anisotropy (defined in terms of $1 / A$ ), with $\alpha=16^{\circ}$, $23^{\circ}$, and $32^{\circ}$ for $\mathrm{Si}, \mathrm{GaAs}$, and $\mathrm{ZnSe}$, respectively. A similar trend is observed for (111) surfaces, i.e., with increasing anisotropy $\alpha$ increases from $19^{\circ}$ for $\mathrm{PbS}$ to $41^{\circ}$ for $\mathrm{SnTe}$.

One can then ask about the consequences for the vertical dot correlations formed during superlattice growth.
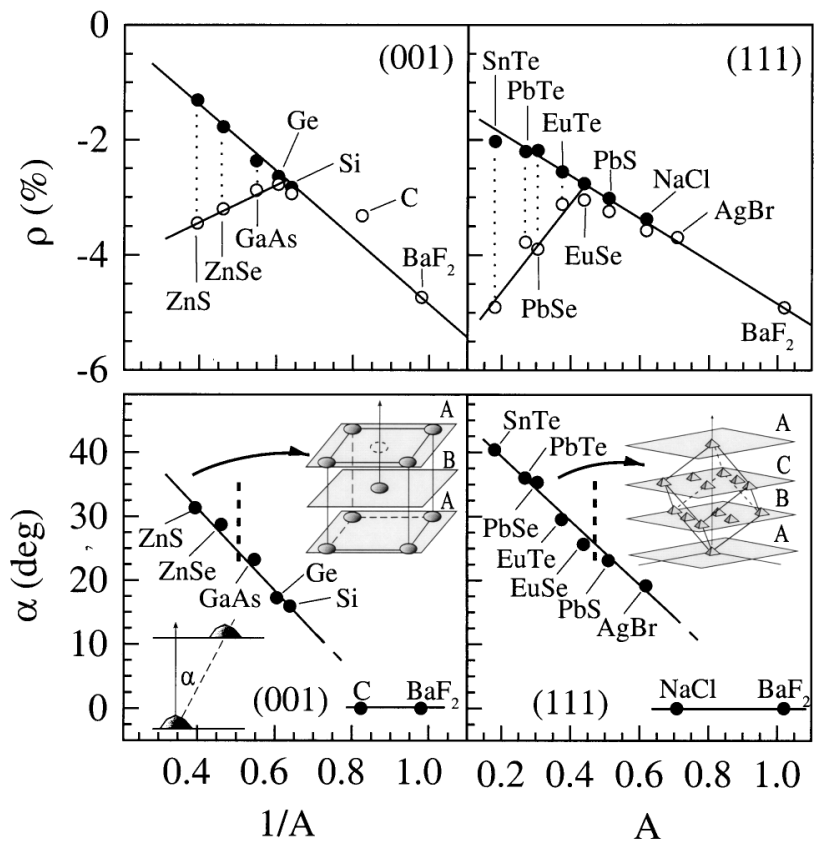

FIG. 2. Upper figures: Normalized energy density in the center above the buried islands $\rho(0)$ and at the side minima positions $\rho_{\min }(\bullet$ and $\circ$, respectively) plotted as a function of elastic anisotropy for (001) and (111) surfaces. Lower figures: angle $\alpha$ relative to the surface normal under which the minima of $\rho(x, y)$ appear on the surface versus elastic anisotropy. Insets: Expected stacking in dot superlattices, centered tetragonal for (001) and $A \geq 1.6$, and trigonal for (111) and $A \leq 0.6$.
For materials and growth orientations with only one central $\rho(x, y)$ minimum, the dots should be vertically aligned, provided that the minima are deep enough to promote preferential growth under given conditions. For other growth orientations, dot correlations along directions inclined to the growth axis are expected. This applies, e.g., for (113) $\mathrm{SiGe} / \mathrm{Si}$ or InAs/GaAs dot superlattices, where the dots should be aligned at about $10^{\circ}$ to the surface normal. If several minima are formed on the surface, however, then these minima also define a preferred lateral dot arrangement. This is clearly most favorable for the formation of 3D ordered dot arrays. For (111) growth and $A \ll 1$, the three subsidiary minima of $\rho$ create a triangle with equally long sides. Therefore, one expects a hexagonal ordering within the growth plane, and a trigonal fcc-like $A B C A B C$ dot stacking sequence in the vertical direction, as shown in the right inset of Fig. 2. For (001) growth and $A \gg 1$, the four side minima define a preferred square arrangement of the dots within the growth plane. Therefore, one may expect an ordering in a tetragonal body centered lattice with an $A B A B$ vertical stacking sequence, as shown in the left inset of Fig. 2.

For the (111) growth direction, the formation of a trigonal dot lattice has indeed been observed for $\mathrm{PbSe} / \mathrm{PbEuTe}$ dot superlattices [9], and the measured trigonal correlation angle of $\alpha=39^{\circ}$ agrees remarkably well with the $36^{\circ}$ derived from our calculations. From Fig. 2, this angle is predicted to vary linearly with $A$ for other IV-VI materials. The situation is more complex for (001) superlattices. For $\mathrm{SiGe} / \mathrm{Si}$ and $\mathrm{InAs} / \mathrm{GaAs}$, experimentally, no centered tetragonal ordering has been observed, but only a simple vertical dot alignment $[2,3,6]$. This is due to the fact that the elastic anisotropy is not sufficiently large in these materials. Thus, the $\rho$ side minima are still very weak, and their lateral spacing is less than the typical island sizes on the surface. In addition, the finite extent of the buried islands will also lead to a smearing of the energy minima. As a result, only one new island will grow on top of a buried island, which is experimentally observed. For the more anisotropic IIVI compounds, the subsidiary minima are about 3 times deeper as for GaAs (see Fig. 1), and indications for a nonvertical dot replication have indeed been reported [7]. Therefore, the II-VI materials seem to be the most suitable candidates for the formation of body-centered tetragonal dot arrangements.

The calculations presented so far imply that for $\mathrm{Si} / \mathrm{Ge}$ or InAs/GaAs (001) superlattices no lateral ordering of the dots should occur. However, experimental studies have indicated that a weak lateral ordering tendency is present along the $\langle 100\rangle$ directions. This can be explained by considering the superposition of $\rho(x, y)$ from adjacent buried islands. As shown in the inset of Fig. 3, for $\mathrm{Si}$ (001) besides the four energy minima near the origin (not shown in this plot), an additional four local $\rho$ maxima 


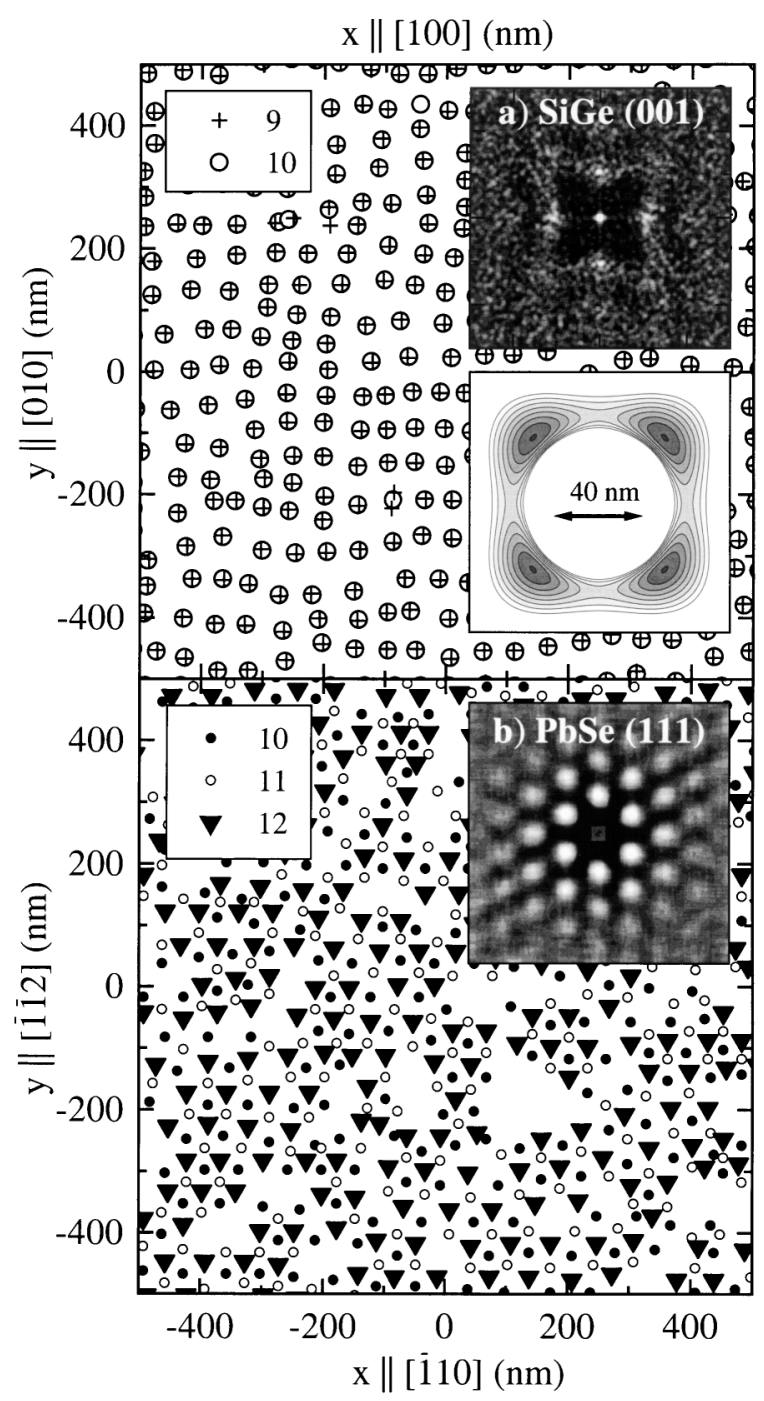

FIG. 3. Growth simulation of the island positions after several superlattice periods: (a) $\mathrm{SiGe}(001)$ islands separated by $20 \mathrm{~nm}$ Si spacer layers after the 9th (+) and 10th (o) layers, (b) PbSe (111) islands separated by $50 \mathrm{~nm}$ PbEuTe spacers after the 10th $(\bullet)$, 11th $(\circ)$, and 12th $(\triangle)$ layers. Insets: 2D Fourier power spectrum of the island positions on the surface. Lower inset in (a): Elastic energy distribution $\rho(x, y)$ above a single $\mathrm{SiGe}$ island. Only positive $\rho$ values are plotted, the regions with $\rho<0$ are not shown (white area in the center). The dark areas are unfavorable for island nucleation.

occur along $\langle 110\rangle$. For a pair of buried dots aligned along $\langle 100\rangle$, the superposition of the strain fields leads to a local $\rho$ minimum in between the dots along the $\langle 110\rangle$ direction. This causes a weak lateral ordering tendency in spite of the absence of pronounced side minima of $\rho$ on the surface.

To prove this phenomenon, we have performed a growth simulation where for each dot layer, we distribute randomly $N=10^{4}$ adatoms on the surface and let them move to the nearest energy minimum. After completion of the layer, we calculate the energy density $\rho(x, y)$ on top of the next wetting layer by summation of the contributions of all islands. Starting from a surface with constant $\rho$, this procedure was repeated several times. In contrast to the isotropic 1D model of Tersoff et al. [4], here we take into account explicitly the elastic anisotropy, and the simulation was performed in two dimensions. For $\mathrm{SiGe} / \mathrm{Si}$ superlattices, the $\mathrm{SiGe}$ dot layers were separated by $20 \mathrm{~nm} \mathrm{Si}$ spacer layers. Figure 3(a) shows the dot positions after the 9th and 10th layers (crosses and circles, respectively). In agreement with the above arguments, we find that the dots are nearly perfectly aligned vertically, with a weak lateral ordering along the $\langle 100\rangle$ directions (see 2D Fourier power spectrum of the dot positions in the upper inset). If we neglect the elastic anisotropy, we do not find any lateral ordering tendency in our simulations. For (111) PbSe/PbEuTe dot superlattices, a similar simulation was carried out. It is emphasized that in this case we find a very effective hexagonal ordering in the lateral direction, and an $A B C A B C$ stacking in the vertical direction [see relative dot positions in the 10th, 11th, and 12th layers in Fig. 3(b)]. This is exactly the dot arrangement observed experimentally [9].

In conclusion, we have demonstrated that the elastic anisotropy is of crucial importance for lateral and vertical self-organization in quantum dot superlattices. Our calculations indicate that a rich diversity in the $3 \mathrm{D}$ dot stacking exists for different materials and growth orientations. The model explains the essential features observed in several multilayer systems, and predicts pronounced 3D ordering for systems with a high elastic anisotropy. This could open the way for fabrication of homogenous dot arrays for device applications.

This work was supported by the FWF, OeNB, and GMe of Austria, and the Grant Agency (20/97/0003) and Ministry of Education of the Czech Republic (VS96192).

*Email address: G.Springholz@hlphys.uni-linz.ac.at

[1] D. Leonard et al., Appl. Phys. Lett. 63, 3203 (1993).

[2] Q. Xie et al., Phys. Rev. Lett. 75, 2542 (1995).

[3] G. S. Solomon et al., Phys. Rev. Lett. 76, 952 (1996).

[4] J. Tersoff, C. Teichert, and M. G. Lagally, Phys. Rev. Lett. 76, 1675 (1996); C. Teichert et al., Phys. Rev. B 53, 16334 (1996).

[5] G.S. Solomon, S. Komarov, J. S. Harris, and Y. Yamamoto, J. Cryst. Growth 175/176, 707 (1997).

[6] V. Holý et al., Phys. Rev. B 58, 7934 (1998).

[7] M. Strassburg et al., Appl. Phys. Lett. 72, 942 (1998).

[8] V.A. Shchukin et al., Phys. Rev. B 57, 12262 (1998).

[9] G. Springholz et al., Science 282, 734 (1998).

[10] J. W. Flocken and J. R. Hardy, Phys. Rev. B 1, 2447 (1970); R. A. Matsumura and G. Sines, J. Appl. Phys. 41, 3930 (1970).

[11] M. A. Krivoglaz, X-Ray and Neutron Scattering in Nonideal Crystals (Springer, Berlin, 1996), Vol. I.

[12] K. Portz and A. Maradudin, Phys. Rev. B 16, 3535 (1977).

[13] C. Pryor et al., J. Appl. Phys. 83, 2548 (1998). 